\title{
Video Article \\ Isometric and Eccentric Force Generation Assessment of Skeletal Muscles Isolated from Murine Models of Muscular Dystrophies
}

\author{
Catherine Moorwood ${ }^{1}$, Min Liu ${ }^{2}$, Zuozhen Tian ${ }^{2}$, Elisabeth R. Barton ${ }^{3}$ \\ ${ }^{1}$ Department of Anatomy and Cell Biology, School of Dental Medicine, University of Pennsylvania \\ ${ }^{2}$ Department of Physiology, Perelman School of Medicine, University of Pennsylvania \\ ${ }^{3}$ Department of Anatomy and Cell Biology, School of Dental Medicine, School of Dental Medicine, University of Pennsylvania
}

Correspondence to: Elisabeth R. Barton at erbarton@dental.upenn.edu

URL: https://www.jove.com/video/50036

DOI: doi: $10.3791 / 50036$

Keywords: Anatomy, Issue 71, Physiology, Cellular Biology, Biophysics, Medicine, Biomedical Engineering, Surgery, Muscles, Muscular Diseases, Animal Experimentation, Chemicals and Drugs, muscular dystrophy, muscle function, muscle damage, muscular dystrophies, mouse, animal model

Date Published: $1 / 31 / 2013$

Citation: Moorwood, C., Liu, M., Tian, Z., Barton, E.R. Isometric and Eccentric Force Generation Assessment of Skeletal Muscles Isolated from Murine Models of Muscular Dystrophies. J. Vis. Exp. (71), e50036, doi:10.3791/50036 (2013).

\section{Abstract}

Critical to the evaluation of potential therapeutics for muscular disease are sensitive and reproducible physiological assessments of muscle function. Because many pre-clinical trials rely on mouse models for these diseases, isolated muscle function has become one of the standards for $\mathrm{Go} / \mathrm{NoGo}$ decisions in moving drug candidates forward into patients. We will demonstrate the preparation of the extensor digitorum longus (EDL) and diaphragm muscles for functional testing, which are the predominant muscles utilized for these studies. The EDL muscle geometry is ideal for isolated muscle preparations, with two easily accessible tendons, and a small size that can be supported by superfusion in a bath. The diaphragm exhibits profound progressive pathology in dystrophic animals, and can serve as a platform for evaluating many potential therapies countering fibrosis, and promoting myofiber stability. Protocols for routine testing, including isometric and eccentric contractions, will be shown. Isometric force provides assessment of strength, and eccentric contractions help to evaluate sarcolemma stability, which is disrupted in many types of muscular dystrophies. Comparisons of the expected results between muscles from wildtype and dystrophic muscles will also be provided. These measures can complement morphological and biochemical measurements of tissue homeostasis, as well as whole animal assessments of muscle function.

\section{Video Link}

The video component of this article can be found at https://www.jove.com/video/50036/

\section{Introduction}

Muscle function measurements contribute to the evaluation of potential treatments for muscle pathology, as well as to the determination of mechanisms underlying physiology of this tissue. For muscle disease, the use of mouse models have become a central component for understanding the links between genotype and phenotype, and for extending that knowledge into the design and testing of potential therapeutics. The muscular dystrophies, in particular, have relied on mice to evaluate these agents and establish pre-clinical data required to move forward to trials in patients. A frequent outcome measure uses isolated muscle function to determine strength, which is applicable to a wide range of studies. Another measure is the use of eccentric, or lengthening, contractions to determine changes in muscle membrane integrity, which is deficient in Duchenne muscular dystrophy, and the mouse model for this disease (mdx). Therefore, it is essential for these types of measurements to be sensitive and reproducible.

The mouse extensor digitorum longus (EDL) muscle has been used extensively for isolated muscle function due to its ideal geometry and size, including uniform fiber orientation and definitive tendons ${ }^{2,5,6,10,12}$. Methods for EDL muscles isometric functional measurements have been described in a previous JoVE publication ${ }^{8}$, as well as in the Treat-NMD SOP ${ }^{1}$. We have extended the description of these methods to include both isometric and eccentric contractions. Hallmarks of disease are evident in the EDL, including heighted cycles of degeneration/regeneration and diminished force output.

The mouse diaphragm exhibits the most rapid pathological progression of muscular dystrophy compared to other muscles in the mouse ${ }^{11}$. By 6 months of age, cumulative fibrosis comprises approximately $50 \%$ of the muscle. This results in significantly impaired force output ${ }^{11}$. Therefore, therapeutic agents that can prevent fibrotic infiltration can be evaluated efficiently in the diaphragm.

The loss of dystrophin in muscle leads to increased fragility and heightened contractile damage in all muscles ${ }^{9}$. Therefore many therapies for Duchenne muscular dystrophy are geared for dystrophin replacement. As such, an assay that has become essential for evaluating these strategies is eccentric contraction, which can distinguish between normal and dystrophic muscle, as well as determine what benefit a particular strategy has for protecting a dystrophic muscle from contractile damage ${ }^{2,3,4,12}$. This procedure requires either a dual-mode servo-motor that can modulate/record length and force, or a method of adjusting length rapidly separate from a force transducer. 


\section{Protocol}

All procedures have been reviewed and approved by University of Pennsylvania IACUC.

\section{EDL Dissection and Preparation (Approximately $30 \mathrm{~min}$ )}

1. Anesthetize mice to ensure that they experience no pain or distress during the procedure, but that the muscles remain well oxygenated by the circulation. We routinely use a Ketamine/xylazine cocktail $(100 / 10 \mathrm{mg} / \mathrm{kg})$ injected via IP. Surgical plane of anesthesia is determined by complete absence of pedal or palpedral withdrawal reflexes, or lack of ear twitch response.

2. Immobilize the hindlimbs using medical tape, and remove the skin of the lower anterior hindlimb to expose the muscles of this area. Keep the muscles moist with the application of PBS at regular intervals.

3. Under a dissecting scope, make a small incision lateral to the knee in order to expose the proximal tendon of the EDL muscle. There are two tendons in this region, and both should be cut to enable removal of the EDL.

4. At the medial ankle, cut the tendon of the TA muscle to expose the distal tendons of the EDL, which extend along the meta tarsals. Lift the TA muscle out of the way, being careful not to cut or touch the EDL underneath. Return to the distal tendons of the EDL, snip each one and draw them through the ankle, then grasping the tendons, gently pull the EDL away from the rest of the limb. It should release from the proximal end freely, but if not, return to the lateral incision to ensure that the tendon is cut. Remove the muscle and place in a dissecting dish filled with chilled oxygenated Ringers. Pin the muscle through the tendons at approximately resting length, which is the length found in vivo. The length is too short when the muscle is flaccid in the dish, and too long if the muscle is pulling on the dissecting pins.

5. Euthanize the mouse immediately following muscle removal by cervical dislocation.

6. Tie sutures to the tendons, as close as possible to the muscle, but not touching the muscle. Pin the muscle at approximately resting length using the sutures.

\section{Diaphragm Dissection and Preparation (Approximately 30 min)}

1. Euthanize the mice prior to this procedure by cervical dislocation under anesthesia, if the same mouse is subjected to both dissections, or by $\mathrm{CO}_{2}$ followed by confirmation with cervical dislocation.

2. Make an incision in the skin to expose the abdominal and chest cavity. Open the abdominal cavity, and cut the body wall just below the ribs. Using bone scissors, and starting above the diaphragm insertion, cut around the entire rib cage following the line of the ribs, and cut through spine. Cut blood vessels running through the center of the diaphragm so that the diaphragm can be removed easily.

3. Remove the diaphragm from the mouse, and place in a dissecting dish filled with oxygenated Ringers. Gently agitate the diaphragm in the dish to wash away blood. Refresh Ringers solution as needed.

4. Cut a small strip of the diaphragm from the central tendon to the ribs along the orientation of the fibers in the central portion of the lateral hemidiaphragms. The strip should be between 2-4 mm wide. Using bone scissors, cut the rib on either side of the strip, leaving approximately 1-2 mm overhang of rib on either side of the diaphragm strip.

5. Tie sutures to the central tendon. Tie suture to each of the laterally protruding rib ends, and then tie these together to make a large loop.

6. Two strips can be obtained from any given diaphragm (one from each side).

\section{Mounting Muscles in Mechanics Bath}

1. Grasp the sutures and use these to attach the muscle to a rigid post on one end, and to a force transducer on the other end.

2. Adjust the length to make sure the muscle is not slack, but isn't taut. A good approximation is the resting muscle length as in $1.4-1.5$.

3. Bath should be filled with oxygenated Ringers solution maintained at $22^{\circ} \mathrm{C}$ to prolong muscle stability for testing. Muscles should rest for 5 $\min$ in this bath prior to functional testing so that muscle temperature is equilibrated to $22^{\circ} \mathrm{C}$.

\section{Isometric Contractions}

1. Establish Supramaximal Stimulation Conditions. After a muscle is placed in the bath, use single 0.5 msec stimulation pulses to generate a twitch, and monitor force output. Gradually increase current until the force attains a maximum but steady level. Increase current to $10 \%$ more than this level for the remaining experiments.

2. Establish Optimum Length. Using isometric twitch stimulations, adjust the length of the muscle gradually until a maximal force is obtained. Rest the muscle $\sim 10 \mathrm{sec}$ between each contraction. Optimal muscle length $\left(\mathrm{L}_{\circ}\right)$ is achieved when twitch force is maximal. Record muscle length (the length between the myotendinous junctions for EDL, and between the central tendon myotendinous junction and the muscle insertion to the rib) using Vernier calipers.

3. Maximum Isometric Tetanic Force. Stimulate muscle set at $\mathrm{L}_{0}$ for a period of $500 \mathrm{msec}$, with a series of 0.5 msec pulses at supramaximal stimulation and at fusion frequency (from the plateau of the frequency-force relationship (e.g. Ref: ${ }^{8}$ ). The plateau for EDL muscles is typically achieved with $120 \mathrm{~Hz}$, and for diaphragm muscles with $100 \mathrm{~Hz}$. Stimulate 3 times at the respective frequency with rest periods of 5 min between stimulation bouts for every muscle.

\section{Eccentric Contractions}

1. Allow muscle to rest 5 min between performing isometric and eccentric contractions

2. Stimulate muscles at $80 \mathrm{~Hz}$ isometrically for the initial $500 \mathrm{msec}$, followed by a $10 \% \mathrm{~L}_{\circ}$ stretch in the final 200 msec stimulation (a stretch rate of $0.5 \mathrm{~L}_{\mathrm{o}} / \mathrm{sec}$ ).

3. Repeat stimulation pattern with 5 min rests between for the desired number of eccentric contractions. 
4. Measure force for each contraction in the time period prior to the stretch. Calculate the drop in force between the first and last contraction.

\section{Muscle Removal from the Apparatus}

1. Shorten the muscle length to allow for gentle removal of the muscle from the transducer and post and return it to a dissecting dish with Ringers.

2. Remove sutures from the muscles.

3. For the EDL muscle, blot the muscle twice, then weigh it, before subsequent processing (e.g. freezing, fixing, etc). This will be important for calculating the cross sectional area, and specific force.

4. For the diaphragm muscle, soak in $0.1 \%$ procion orange, a membrane impermeant dye for $15-20$ min. This will provide an index of dissection damage.

5. Dissect the muscle away from the bony insertion, as well as the central tendon. This is necessary to provide an accurate weight of the muscle. Blot as above before weighing and subsequent preparation.

6. Calculate Cross Sectional Area (CSA):

CSA $\left(\mathrm{mm}^{2}\right)=\operatorname{mass}(\mathrm{mg}) /\left[\left(\mathrm{L}_{\mathrm{o}} \mathrm{mm}\right) *\left(\mathrm{~L} / \mathrm{L}_{\mathrm{o}}\right) *\left(1.06 \mathrm{mg} / \mathrm{mm}^{3}\right)\right]$

where $L / L_{o}$ is the fiber to muscle length ratio $(0.45 \text { for } E D L, 1.0 \text { for the diaphragm })^{5}$ and 1.06 is the density of muscle.

\section{Representative Results}

Expected values for the isometric forces of EDL muscles from 12 week old animals are shown in Figure 1. Because mdx muscles exhibit compensatory hypertrophy, total tetanic force can be higher in $\mathrm{mdx}$ muscle compared to age matched wildtype controls. However, a more appropriate measure of functional output is specific force (Figure 2), where force is normalized for cross-sectional area to calculate this value. Specific force depends on the inherent functional capacity of the muscle, where the weakness in dystrophic muscles is more apparent when both absolute force and cross sectional area are accounted for. In our hands, EDL muscles from mdx mice generate approximately 20 - $25 \%$ lower specific forces than those of age matched wildtype mice from $10-26$ weeks of age.

For the diaphragm, only specific force is relevant for comparisons because the preparation is a piece of the muscle dependent upon the dissection. Comparison of specific force between wildtype and $\mathrm{mdx}$ diaphragm muscles reflects the progressive pathology in this tissue. Isometric force output diminishes with age (Figure 3), so that by 6 months of age, diaphragm muscles from mdx mice produce no more than half of the functional output of diaphragm strips from age-matched wildtype controls.

An example of eccentric contractions from diaphragm testing is shown in Figure 4. With each subsequent eccentric contraction, force output diminishes in diaphragm strips from both wildtype (C57) and mdx mice. However, the loss of force is more dramatic in muscle samples from the $\mathrm{mdx}$ mouse, presumably from the absence of dystrophin and its associated proteins.

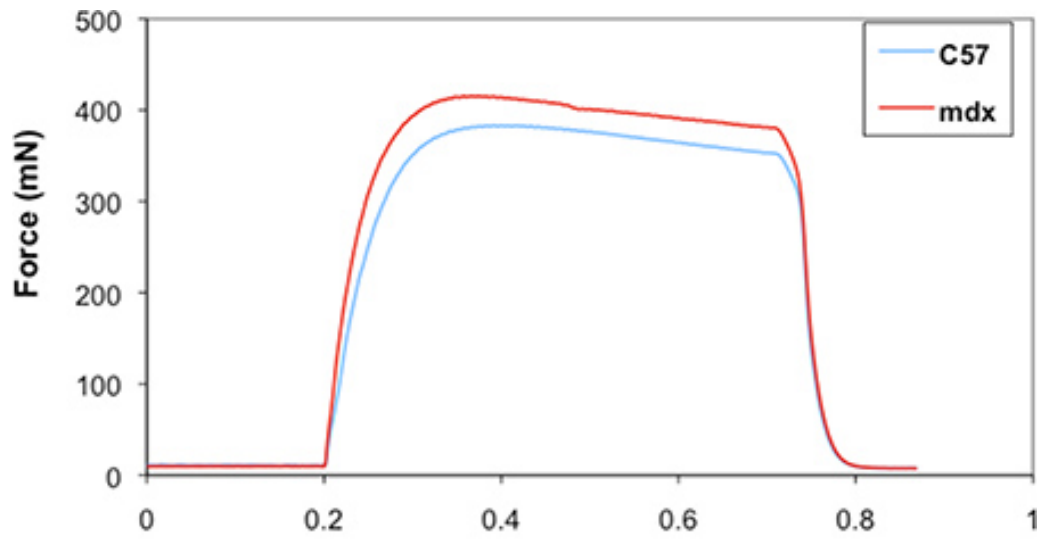

Time (sec)

Figure 1. Isometric tetanic force in wildtype and mdx EDL muscles from 12 week old mice. Absolute force can be higher in age-matched mdx EDL muscles due to compensatory hypertrophy. 


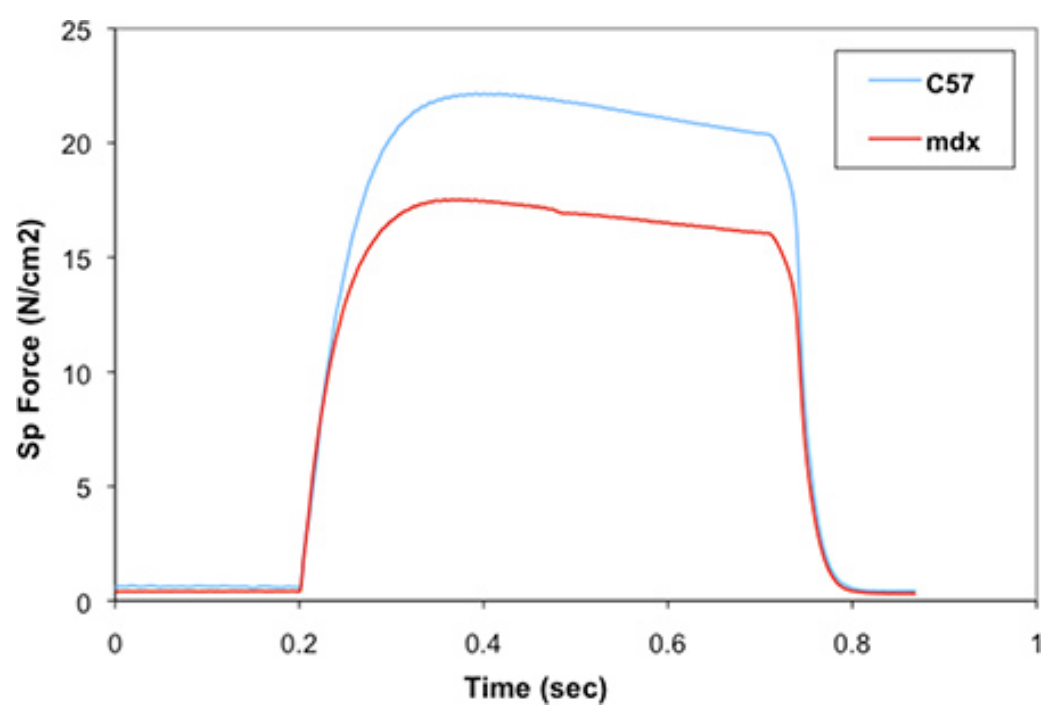

Figure 2. Isometric tetanic force normalized by muscle cross sectional area is Specific Force, and reveals depressed functional capacity of EDL muscles from $m d x$ mice compared to those of wildtype mice. Traces are from the same mice as in Figure 1.

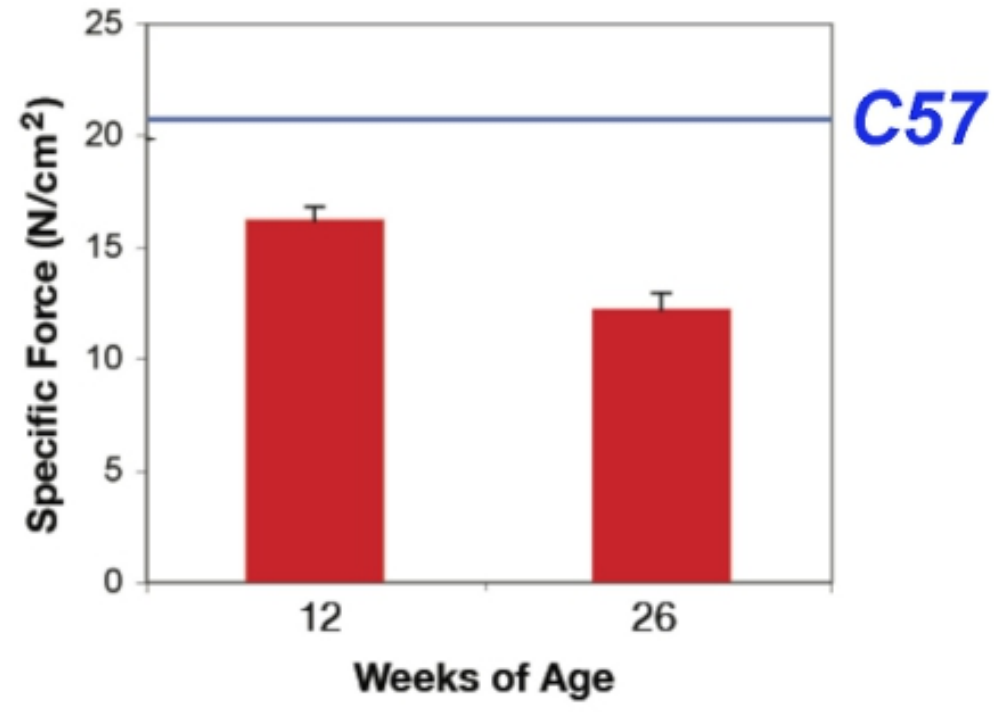

Figure 3. The progressive loss of force generating capacity assessed by specific force is most apparent in the diaphragm of mdx mice (red bars) compared to wildtype (C57 in blue line). 


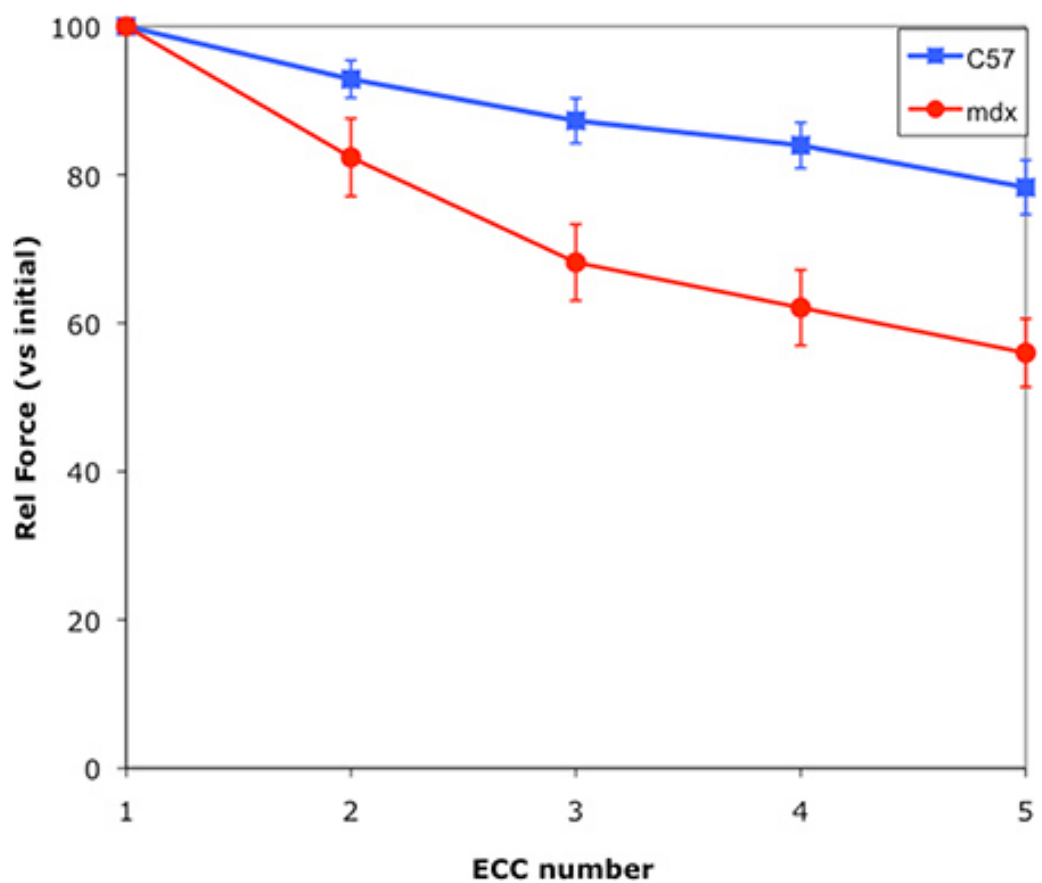

Figure 4. The loss of force as a function of eccentric contraction number for diaphragm muscles from 12 week old C57 and mdx mice. Data is mean \pm SEM for $\mathrm{N}=4$ muscles per genotype.

\section{Discussion}

The goal of this article is to provide guidance for performing isolated muscle function on two muscles from mice - the EDL and the diaphragm. Evaluation of these muscles can provide insight into whether or not therapeutic candidates for muscle disease are beneficial. For both muscles, the major factor in obtaining robust data is a clean dissection. Therefore, practicing and perfecting the initial isolation step is essential before moving onto functional testing. In addition, establishing functional benchmarks for normal muscle is critical prior to making comparisons to dystrophic muscle, or between treatments. This will assure that the study results are not subject to high variability imparted by the ability of the person performing the experiments. The use of a membrane impermeant dye can help with optimizing the dissection preparation, for incubation of any muscle in a solution containing such a dye will mark most damaged fibers, and can serve as an index of dissection success. Minimizing the number of fibers damaged in the muscle will help to optimize the measurement. The fibers damaged by dissection fluoresce much more brightly than those damaged by eccentric contraction, and so if the dye is also used during the mechanics process, one can use the intensity of dye to distinguish between the two types of damage.

Dissection of diaphragm strips will almost always have fiber damage, simply because by cutting along the length of the fibers, some inevitably get destroyed. Dye uptake is strong in those damaged fibers, and these are normally restricted to the outer edges of the preparation. On average, we observe a band of damaged fibers that is $\sim 3$ fibers in width $(\sim 120 \mu \mathrm{m})$ on either side of the muscle strip. If the damaged band comprises more than $15 \%$ of the muscle, then the data is discarded. The diaphragm preparation also has constraints on the optimum size for functional measures. We have found that pieces of diaphragm that are wider than $5 \mathrm{~mm}$ begin to fold up on themselves, because the central tendon tie is only at one point. This results in decreased specific force in the preparation. We have also found that narrower strips also have lower specific force, which we believe is because the numbers of fibers damaged during dissection comprise a larger proportion of the total fiber number. For instance, if $0.1 \mathrm{~mm}$ on each side is damaged, then that is $5 \%$ (2x0.1 $\mathrm{mm} / 4 \mathrm{~mm}$ strip) of the muscle preparation that doesn't contribute to force, whereas if the strip is only $1 \mathrm{~mm}$, then $20 \%$ of the muscle preparation is damaged. Thus, both the width of the damaged region as well as the width of the entire preparation are important factors to control.

Measurements of maximum isometric tension require that all muscle fibers in a muscle are stimulated. Because there is tremendous variability in the components of a function apparatus, this must be determined for each individual set up. For example, bath size or the type of stimulator can affect the intensity of stimulation. Twitch stimulation is a reasonable way to determine supramaximal stimulation conditions. Once this is established for a specific setup and muscle, it can be utilized for subsequent studies.

In contrast, the optimum length of any given muscle needs to be measured for each preparation using the iterative process described above. This ensures that thick and thin filament overlap is optimal and the maximum potential force generating capacity is measured. Alternative procedures can rely on the diffraction patterns associated with the muscle striations, but this requires additional equipment not described here.

We routinely use $500 \mathrm{msec}$ stimulation durations, which falls in the middle range of this parameter used by other investigators in this field. Although this might cause some fatigue of the muscle during the contraction, which is evident by a "sag" in the maximal force production, this in itself can be informative. For instance, a difference in fatigue could be achieved by different types of therapies including those that target calcium handling, hence the sag in force during active contraction can serve as an index for improvement. Alternatively, the loss of force could indicate that the sutures on the tendons are not tight enough, and that they are slipping during the contraction. The muscle must be removed from the bath and the sutures must be re-tied if this occurs. We also use a series of 3 tetanic contractions, which helps evaluate the stability of the preparation. Again, suture slips would lead to loss of force between contractions, requiring suture re-tying. Large muscles may also 
generate anoxic cores, which lead to losses of force during the protocol. Muscle size is a limiting factor for performing isolated muscle function testing, where EDL muscles with masses greater than $20 \mathrm{mg}$ lose force with each contraction, and cannot be supported by superfusion in a bath. Diaphragm strips do not suffer from the same complications because they are thin enough to have prolonged viability in the bath.

Other muscles can be utilized for isolated muscle function, including the soleus muscle, which is commonly used, but not described here. Many of the same procedures can be adopted for the soleus in terms of the preparation and the functional testing. However, the main differences are in the stimulation frequency, and the parameters for eccentric contractions. Use of the soleus for function complements that of the other two muscles, and so it should be considered as part of a "standard package " for evaluating dystrophic mice ${ }^{4,7}$.

Eccentric contraction provides an index of contractile fragility, and it is important to use a protocol that results in modest loss of force in muscles from wildtype animals and a significant loss of force in untreated dystrophic muscles so that there is a wide dynamic range for comparisons. A lack of any force loss in normal muscles suggests that the eccentric contraction is too mild, and will not be adequate to distinguish between therapies that are not effective and those that are truly beneficial. However, a dramatic loss of force in normal muscles subjected to eccentric contraction may be too great to tease out differences associated with the disease. Our protocol for the EDL and diaphragm uses a $0.5 \mathrm{Lo} /$ sec stretch rate to produce a $10 \%$ length change. We typically perform 5 eccentric contractions, which result in a small loss of force in normal muscles and a significant loss of force in dystrophic muscles. Certainly, all of these parameters can be varied to increase the overall length change, the rate of stretch, or the number of eccentric contractions in order to distinguish differences between diseased and healthy muscle, as well as on the effects of a specific type of mutation or treatment one is studying. As long as there is a marked difference between normal and diseased muscles, then there is a gold standard to reach for in terms of treatments.

In summary, this protocol establishes guidelines for performing isometric and eccentric contractions, and hopefully identifies the potential pitfalls to avoid when setting this technique up in your lab.

\section{Disclosures}

Production and Free Access to this article is sponsored by Aurora Scientific.

\section{Acknowledgements}

This work was supported by the Paul D. Wellstone Cooperative Research Center (AR052646).

\section{References}

1. Barton, E.R., Khurana, T.S., \& Lynch, G.S. Measuring isometric force of isolated mouse muscles in vitro. TREAT-NMD SOP Number M.1.2 002 (2008).

2. Barton, E.R., Morris, L., Musaro, A., Rosenthal, N., \& Sweeney, H.L. Muscle-specific expression of insulin-like growth factor I counters muscle decline in mdx mice. J. Cell Biol. 157, 137-148 (2002).

3. Barton, E.R., Morris, L., Kawana, M., Bish, L.T., \& Toursel, T. Systemic administration of L-arginine benefits mdx skeletal muscle function. Muscle Nerve. 32, 751-760 (2005).

4. Barton, E.R., Wang, B.J., Brisson, B.K., \& Sweeney, H.L. Diaphragm displays early and progressive functional deficits in dysferlin-deficient mice. Muscle Nerve. 42 (1), 22-9 (2010).

5. Brooks, S.V. \& Faulkner, J.A. Contractile properties of skeletal muscles from young, adult and aged mice. J. Physiol. 404, 71-82 (1988).

6. Harcourt, L.J., Schertzer, J.D., Ryall, J.G., \& Lynch, G.S. Low dose formoterol administration improves muscle function in dystrophic mdx mice without increasing fatigue. Neuromuscul. Disord. 17(1), 47-55 (2007).

7. Lynch, G.S., Hinkle, R.T., Chamberlain, J.S., Brooks, S.V., \& Faulkner, J.A. Force and power output of fast and slow skeletal muscles from mdx mice 6-28 months old. J. Physiol. 535 (Pt. 2), 591-600 (2001).

8. Oishi, P.E., Cholsiripunlert, S., Gong, W., Baker, A.J., \& Bernstein, H.S. Myo-mechanical Analysis of Isolated Skeletal Muscle. J. Vis. Exp. (48), e2582, doi:10.3791/2582 (2011).

9. Petrof, B.J., Shrager, J.B., Stedman, H.H., Kelly, A.M., \& Sweeney, H.L. Dystrophin protects the sarcolemma from stresses developed during muscle contraction. Proc. Natl. Acad. Sci. U.S.A. 90, 3710-3714 (1993).

10. Salomonsson, S., Grundtman, C., Zhang, S.J., Lanner, J.T., Li, C., Katz, A., Wedderburn, L.R., Nagaraju, K., Lundberg, I.E., \& Westerblad, $\mathrm{H}$. Upregulation of $\mathrm{MHC}$ class I in transgenic mice results in reduced force-generating capacity in slow-twitch muscle. Muscle Nerve. 39 (5), 674-82 (2009).

11. Stedman, H.H., Sweeney, H.L., Shrager, J.B., Maguire, H.C., Panettieri, R.A., Petrof, B., Narusawa, M., Leferovich, J.M., Sladky, J.T., \& Kelly AM. The mdx mouse diaphragm reproduces the degenerative changes of Duchenne muscular dystrophy. Nature. 352, 536-539 (1991).

12. Welch, E.M., et al. PTC124 targets genetic disorders caused by nonsense mutations. Nature. 447 (7140), 87-91 (2007). 